\title{
EVALUACIÓN DE CRECIMIENTO Y FORMA DE FUSTE DE UN ENSAYO DE PROCEDENCIAS Y PROGENIES DE COIHUE (Nothofagus dombeyi (Mirb.) Oerst.) DE 15 AÑOS DE EDAD
}

\author{
Gutiérrez, Braulio ${ }^{11}$
}

\section{RESUMEN}

Se evalúa el crecimiento y la forma del fuste en un ensayo de Nothofagus dombeyi de 15 años de edad establecido en el predio Huillilemu (San José de la Mariquina, región de Los Ríos). Se constata una deficiente forma de fuste expresada en una alta incidencia de bifurcaciones y dobles flechas que coincide con la información indicada en la bibliografía. Esta característica no muestra diferencias entre zonas de procedencia ni entre familias indicando que sería una característica propia de la especie.

Los crecimientos en altura y diámetro, expresados en incrementos medios anuales, son superiores a los registrados en renovales de coinue y similares a los mencionados para plantaciones de edad equivalente a la del ensayo evaluado.

Al contrario que la forma, los crecimientos muestran diferencias significativas entre algunas familias, aunque la mayoría de ellas no evidencian diferencias para las variables de crecimiento. Tampoco se registran diferencias de crecimiento entre zonas de procedencia.

Palabras clave: Nothofagus dombeyi, crecimiento, forma, procedencias, progenies

\section{SUMMARY}

The growth and the stem shape of Nothofagus dombeyi were evaluated in a 15 years old provenance progeny trial established in the Huillilemu farm (San José de la Mariquina, Los Ríos region). Such is indicated in bibliography, a poor stem shape was observed, expressed in a high incidence of bifurcations and double arrows stems. This trait does not show differences between provenance zones or between families, indicating that it would be a common characteristic of the species.

The growths in height and diameter, expressed in annual average increments, are higher than those recorded in Coihue second growth stands and similar to those mentioned for plantations of an age equivalent to that of the evaluated trial.

On the contrary that the form, the growths show significant differences between some families, although most of them do not show differences for the growth variables. There are also no growth differences between provenance zones.

Keywords: Nothofagus dombeyi, growth, stem shape, provenances, progenies

\footnotetext{
${ }^{11}$ Ingeniero Forestal. Instituto Forestal, Sede Bio Bio. bgutierr@infor.cl
} 


\section{INTRODUCCIÓN}

Dentro de las especies nativas que se presentan en plantaciones forestales, coihue ha sido una de la más difundidas debido a su atractiva tasa de crecimiento. Plantaciones con silvicultura intensiva de establecimiento pueden alcanzar incrementos medios anuales de hasta 1,9 cm en diámetro y $1,5 \mathrm{~m}$ en altura (Donoso y Soto, 2010).

No obstante, en estas plantaciones es común observar árboles con mala forma de fuste, bifurcaciones recurrentes, torceduras e inserción de ramas muertas que perjudican la calidad y rendimiento de la producción maderera.

En efecto, en coihue es común que las yemas terminales aborten por falta de protección, lo que provoca que el tallo se construya a partir de yemas laterales, lo que tiende a aumentar las bifurcaciones del fuste (Cruz-Johnson, 2013).

Esta situación es reconocida como uno de los principales problemas de malformación que presenta la especie, y que la hace tender a bifurcarse y formar doble flecha (doble ápice) desde el inicio de su crecimiento (Betancurt, 2015).

Otros autores también señalan que coihue se bifurca tempranamente, incluso después de aplicarle podas de formación o de corrección fustal (Donoso et al., 1999; Viñuales, 2012).

Por tal motivo, es especialmente interesante generar información respecto al desempeño de coihue en plantaciones, analizar su comportamiento a nivel de procedencias y progenies de modo de disponer de antecedentes para orientar programas de mejoramiento genético que contribuyan a aumentar su rendimiento y reducir la incidencia de defectos que disminuyen su productividad.

Consecuentemente, en el presente artículo se evalúa un ensayo de procedencias y progenies de coihue, con el objeto de caracterizar su crecimiento y forma de fuste a nivel de progenies y zonas de procedencia.

\section{MATERIAL Y MÉTODO}

El ensayo considerado corresponde a una prueba de procedencias y progenies de coigüe establecido en el predio Huillilemu el año 2002. El ensayo considera 23 procedencias y 73 progenies distribuidas en 5 bloques al azar con parcelas STP ${ }^{12}$.

La medición se efectuó en mayo de 2017, ocasión en que se registró para cada uno de los individuos del ensayo sus valores de altura (medida en metros, con un hipsómetro Vertex y una precisión de $0,1 \mathrm{~m}$ ); diámetro a 1,3 metros (medido en centímetros, con huincha diamétrica estándar y una precisión de $0,1 \mathrm{~cm}$ ); rectitud de fuste (evaluada con escala cualitativa de 4 categorías, Figura $\left.\mathrm{N}^{\circ} 1\right)$; y observaciones respecto a hábito del fuste $\left(\mathrm{N}^{\circ}\right.$ y altura de bifurcaciones y presencia de multiflechas).

\footnotetext{
${ }^{12}$ Single tree plot: un árbol por parcela 
Con los datos de la medición se confeccionó una base de datos, la que se utilizó para evaluar y analizar el ensayo en términos de crecimiento y forma/calidad de fuste. Las variables analizadas correspondieron a:

Altura: Altura total desde el suelo hasta el ápice del árbol

DAP: Diámetro a altura del pecho (1,3m), en el caso de árboles bifurcados o trifurcados que presentan más de un fuste, se utilizó el DAP equivalente, que corresponde a un diámetro teórico que representa la misma área basal que la suma de los fustes que lo componen.

Rectitud de Fuste: Evaluada con una escala de cuatro categorías (Figura $N^{\circ} 1$ )

Calidad de Fuste: Índice construido para cada árbol a partir de los datos de rectitud de fuste y de las observaciones de bifurcaciones y multiflechas. Se asignan puntajes por rectitud, altura de bifurcación y presencia o ausencia de multiflechas. La suma de los puntajes en cada variable se transforma a una escala porcentual (0-100) y se transforma a unidades de Bliss ${ }^{13}$ para efectos del análisis estadístico.

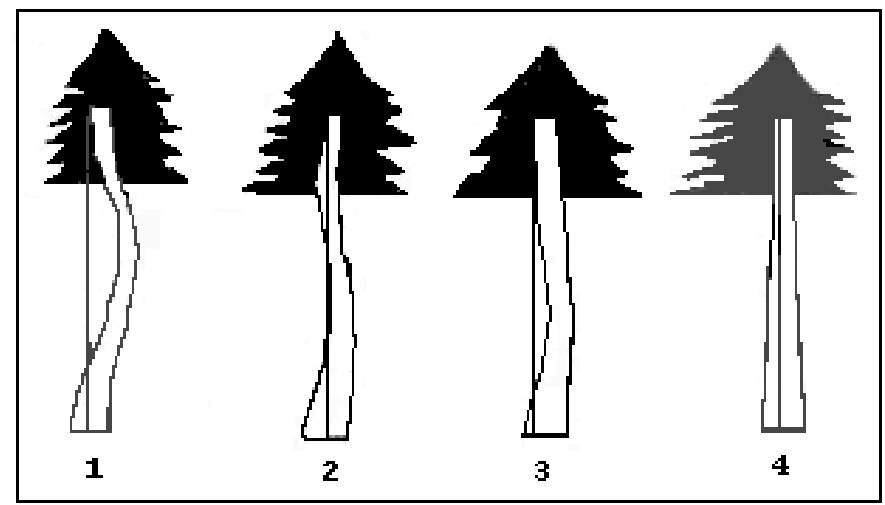

Figura $\mathrm{N}^{\circ} 1$

ESCALA CUALITATIVA PARA EVALUCIÓN DE RECTITUD DE FUSTE

Se calcularon los parámetros medios y de dispersión del ensayo para cada variable evaluada y se efectuó análisis de varianza para verificar la existencia de diferencias estadísticamente significativas para cada una de las variables a nivel de zonas de procedencia, y progenies $^{14}$. Para el análisis a nivel de zonas de procedencia, se clasificó a cada una de ellas en la zona correspondiente de acuerdo a la definición de Quiroz y Gutiérrez (2014), la que se ilustra en la Figura $\mathrm{N}^{\circ} 2$.

\footnotetext{
${ }^{13}$ La transformación de Bliss se utiliza para efectuar análisis de varianza de variables expresadas en porcentaje. Corresponde a la raíz cuadrada del arcoseno del porcentaje expresado en tanto por uno: [U Bliss = raíz (arc sen $(\% / 100))]$

${ }^{14}$ Conjunto de individuos que provienen de un árbol madre común. Entre ellos son medio-hermanos (halfsibs)
} 


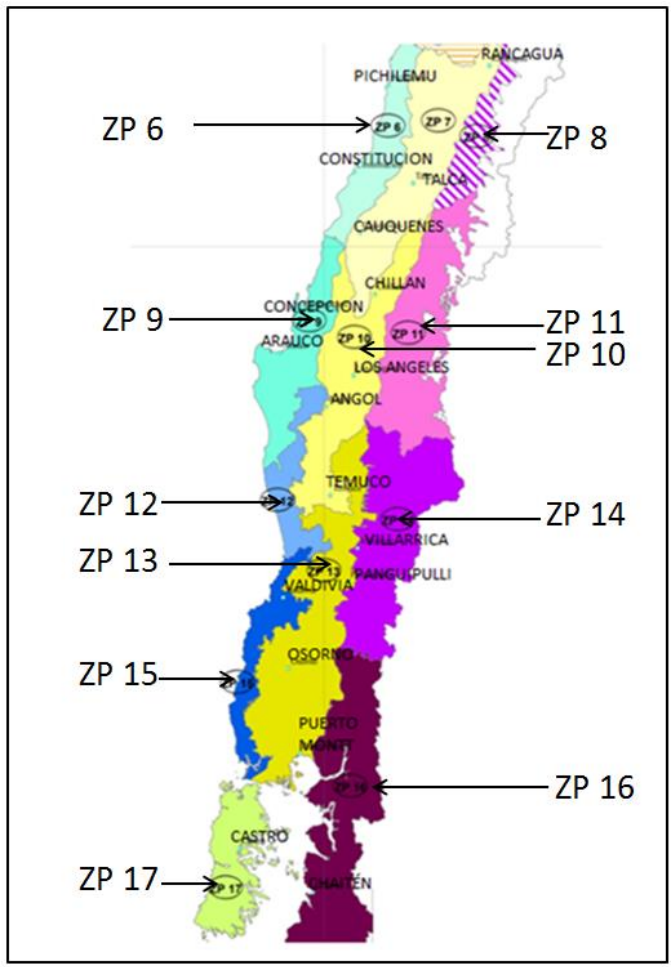

Figura $\mathbf{N}^{\circ} 2$

DEFINICIÓN DE ZONAS DE PROCEDENCIAS UTILIZADA PARA INDIVIDUALIZAR LAS PROCEDENCIAS EVALUADAS EN EL ENSAYO

Los análisis de varianza se complementaron con la prueba de Tuckey para comparación múltiple de medias (alfa=0,05), usando la diferencia mínima significativa (DMS) como criterio para decidir la existencia de diferencias estadísticamente significativas para cada variable en función de las zonas de procedencia y las progenies.

Para efectos de contrastar los parámetros de crecimiento con otros reportados en la bibliografía se utilizó el Incremento Medio Anual (IMA) de altura y DAP.

Respecto a forma y calidad de fuste, las comparaciones se efectuaron en términos de porcentaje de incidencia de bifurcaciones, por cuanto esta es la forma en que esta variable se representa en la bibliografía. 


\section{RESULTADOS}

\section{Crecimiento y Supervivencia}

Los resultados de la medición indican que a los 15 años de edad, el ensayo posee una supervivencia de $72 \%$, presenta una altura promedio de $15,4 \mathrm{~m}$ y un DAP de $18 \mathrm{~cm}$ (Cuadro $\mathrm{N}^{\circ} 1$ ). Estos crecimientos corresponden a incrementos medios anuales (IMA) de 1,03 m para la altura y de $1,2 \mathrm{~cm}$ para el DAP.

Cuadro $\mathrm{N}^{\circ} 1$

ESTADISTICAS DESCRIPTIVAS DEL ENSAYO

\begin{tabular}{|l|r|r|r|}
\cline { 2 - 4 } \multicolumn{1}{c|}{} & \multicolumn{1}{c|}{$\begin{array}{c}\text { Supervivencia } \\
\text { (\%) }\end{array}$} & \multicolumn{1}{c|}{$\begin{array}{c}\text { Altura }^{2} \\
\text { (m) }\end{array}$} & \multicolumn{1}{c|}{$\begin{array}{c}\text { DAP } \\
\text { (cm) }\end{array}$} \\
\hline Promedio & $\mathbf{7 1 , 8}$ & $\mathbf{1 5 , 4}$ & $\mathbf{1 8 , 0}$ \\
\hline VMAX & 100,0 & 26,7 & 32,5 \\
\hline VMIN & 20,0 & 7,3 & 6,8 \\
\hline Desviación Estándar & 22,9 & 2,5 & 4,3 \\
\hline
\end{tabular}

1: Promedios por progenie 2: Promedios por árbol

A nivel de zonas de procedencia, los valores medios de altura y DAP se presentan en el Cuadro $\mathrm{N}^{\circ} 2$. Considerando que la prueba de Tuckey (alfa $=0,05$ ) determinó como diferencia mínimas significativas de $4,02 \mathrm{~m}$ para la altura y de $6,93 \mathrm{~cm}$ para el DAP, se observa que no existen diferencias estadísticamente significativas entre zonas de crecimiento para ninguna de las dos variables de crecimiento evaluadas (altura y DAP).

\section{Cuadro $\mathrm{N}^{\circ} 2$}

VALORES MEDIOS DE ALTURA Y DAP POR ZONA DE PROCEDENCIAS Y DIFERENCIA MÍNIMA SIGNIFICATIVA (DMS)

\begin{tabular}{|c|rc|rc|}
\hline $\begin{array}{c}\text { Zonas de } \\
\text { Procedencia }\end{array}$ & $\begin{array}{c}\text { Altura } \\
(\mathbf{m})\end{array}$ & $\begin{array}{c}\text { DAP } \\
(\mathbf{c m})\end{array}$ \\
\hline ZP 6 & 15,66 & a & 18,62 & a \\
\hline ZP 8 & 13,71 & a & 15,00 & a \\
\hline ZP 9 & 16,61 & a & 18,82 & a \\
\hline ZP10 & 14,20 & a & 14,91 & a \\
\hline ZP11 & 15,08 & a & 17,89 & a \\
\hline ZP12 & 15,75 & a & 17,93 & a \\
\hline ZP13 & 16,24 & a & 18,04 & a \\
\hline ZP14 & 16,79 & a & 17,95 & a \\
\hline ZP15 & 15,47 & a & 19,50 & a \\
\hline ZP16 & 16,39 & a & 19,21 & a \\
\hline ZP17 & 14,20 & a & 14,94 & a \\
\hline Promedio & $\mathbf{1 5 , 4 1}$ & & $\mathbf{1 8 , 0 2}$ & \\
\hline DMS (Tuckey alfa $=\mathbf{0 , 0 5 )}$ & $\mathbf{4 , 0 2}$ & $\mathbf{6 , 9 3}$ & \\
\hline
\end{tabular}

A nivel de progenies, tanto para la altura como para el diámetro, se distinguen dos grupos estadísticamente distintos, con alto nivel de traslape, que se resumen en el Cuadro №3. 
Cuadro $\mathrm{N}^{\circ} 3$

VALORES MEDIOS DE ALTURA Y DAP POR PROGENIE Y DIFERENCIA MÍNIMA SIGNIFICATIVA (DMS)

\begin{tabular}{|c|c|}
\hline Progenie & $\begin{array}{c}\text { Altura } \\
(\mathbf{m})\end{array}$ \\
\hline 217 & $11,23 \quad \mathrm{a}$ \\
\hline Grupo de 71 progenies & $12,05-18,85 \quad$ ab \\
\hline 142 & $20,00 \quad \mathrm{~b}$ \\
\hline Promedio & $\mathbf{1 5 , 4 1}$ \\
\hline DMS (Tuckey alfa $=\mathbf{0 , 0 5})$ & $\mathbf{8 , 4 5}$ \\
\hline
\end{tabular}

\begin{tabular}{|c|r|}
\hline Progenie & $\begin{array}{c}\text { Altura } \\
(\mathbf{m})\end{array}$ \\
\hline 251 & $8,3 \quad \mathrm{a}$ \\
\hline Grupo de 69 progenies & $11,29-22,42 \mathrm{ab}$ \\
\hline 261 & $23,22 \quad \mathrm{~b}$ \\
\hline 142 & $24,19 \mathrm{~b}$ \\
\hline 373 & $24,30 \mathrm{~b}$ \\
\hline Promedio & 18,02 \\
\hline DMS (Tuckey alfa $=\mathbf{0 , 0 5 )}$ & $\mathbf{1 4 , 5 4}$ \\
\hline
\end{tabular}

\section{Forma de Fuste}

La evaluación del ensayo permitió constatar una alta incidencia de árboles bifurcados bajo y sobre el DAP, así como también una abundante presencia de individuos de crecimiento simpódico (bifurcaciones recurrentes y multiflecha) donde no se distingue un fuste principal. Solo el $52,2 \%$ de los árboles exhibe un hábito de crecimiento monopódico, mientras que los restantes presentan bifurcaciones y múltiples flechas en las proporciones que se indican en el Cuadro $\mathrm{N}^{\circ} 4$.

\section{Cuadro $\mathrm{N}^{\circ} 4$}

INCIDENCIA DE BIFURCACIONES Y MULTIPLES FLECHAS EN LOS FUSTES

\begin{tabular}{|l|l|r|r|r|}
\cline { 3 - 4 } \multicolumn{2}{c|}{} & \multicolumn{2}{c|}{$\begin{array}{c}\text { Multiflecha } \\
(\%)\end{array}$} & \multicolumn{1}{c|}{$\begin{array}{c}\text { Subtotal } \\
(\%)\end{array}$} \\
\cline { 3 - 4 } \multicolumn{2}{c|}{} & \multicolumn{1}{c|}{ No } & \multicolumn{1}{c|}{ Si } & \multicolumn{1}{c|}{} \\
\hline \multirow{3}{*}{ Bifurcados } & Bajo DAP & 7,6 & 7,3 & $\mathbf{1 4 , 9}$ \\
\cline { 2 - 5 } & Sobre DAP & 15,3 & 17,6 & $\mathbf{3 2 , 9}$ \\
\cline { 2 - 5 } & Subtotal & $\mathbf{2 2 , 9}$ & $\mathbf{2 4 , 9}$ & $\mathbf{4 7 , 8}$ \\
\hline Monopódicos & 52,2 & & 52,2 \\
\hline \multicolumn{2}{|l|}{ Total } & $\mathbf{7 5 , 1}$ & $\mathbf{2 4 , 9}$ & $\begin{array}{r}\mathbf{1 0 0 , 0} \\
(\mathbf{n = 3 2 3})\end{array}$ \\
\hline
\end{tabular}

La distribución porcentual de la rectitud de fuste, bifurcaciones, multiflechas y calidad de fuste se presenta en la Figura $\mathrm{N}^{\circ} 3$, donde se aprecia una alta concentración de los individuos en la categoría más baja de rectitud (más torcidos) con un $65,9 \%$ y una mínima presencia de árboles rectos; respecto a las bifurcaciones, estas se presentan en el $47,8 \%$ de los árboles y cerca del $25 \%$ de ellos presentan multiflecha. En cuanto a la calidad del fuste, variable que reúne información de rectitud, bifurcaciones y multiflechas, el $82,4 \%$ de los individuos se concentra en valores de calidad menores a $50 \%$.

En promedio la rectitud de fuste alcanzó un valor de 1,37 en una escala de 1 a 4 , en tanto que la calidad del fuste alcanzó a 30,56\% en una escala porcentual (donde 0 es lo peor y 100 lo mejor). Los valores obtenidos ilustran una pobre forma y calidad de los fustes de coihue, situación que como se analizará posteriormente en la sección de discusión de resultados, es coincidente con información publicada por diversos autores que han analizado estas mismas variables en otras plantaciones de esta especie. 


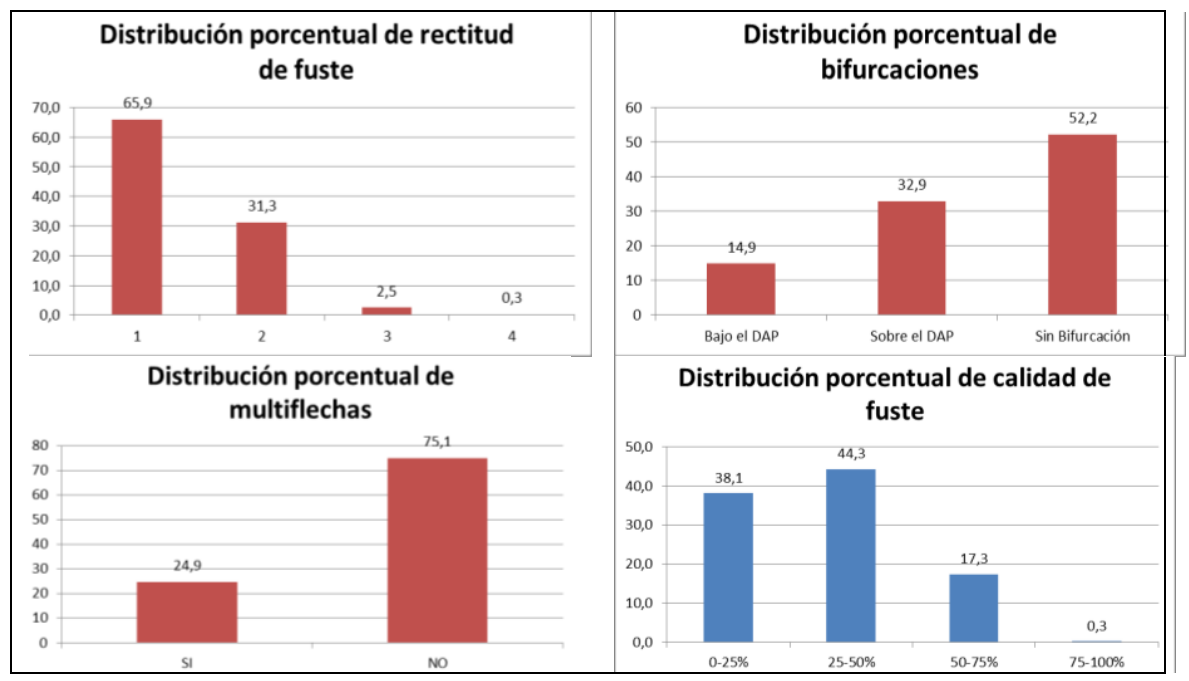

Figura $\mathbf{N}^{\circ} 3$

DISTRIBUCIÓN DE FRECUENCIAS RELATIVAS DE RECTITUD DE FUSTE, BIFURCACIONES, MULTIFLECHAS Y CALIDAD DE FUSTE (COMO COMBINACIÓN DE LAS ANTERIORES)

En los análisis de varianza efectuados, la rectitud y calidad de fuste no presentó diferencias estadísticamente significativas entre zonas de procedencias (Cuadro $N^{\circ} 5$ ) ni entre progenies (Cuadros $\mathrm{N}^{\circ} 6$ ).

\section{Cuadro $\mathrm{N}^{\circ} 5$}

VALORES MEDIOS DE RECTITUD Y CALIDAD DE FUSTE POR ZONAS DE PROCEDENCIA Y DIFERENCIA MÍNIMA SIGNIFICATIVA (DMS)

\begin{tabular}{|c|cc|cc|}
\hline Zonas de Procedencia & \multicolumn{2}{|c|}{$\begin{array}{c}\text { Rectitud Fuste } \\
\text { (1-4) }\end{array}$} & \multicolumn{2}{c|}{$\begin{array}{c}\text { Calidad Fuste } \\
\text { (\%) }\end{array}$} \\
\hline ZP 6 & 1,26 & $\mathrm{a}$ & 33,23 & $\mathrm{a}$ \\
\hline ZP 8 & 1,53 & $\mathrm{a}$ & 33,33 & $\mathrm{a}$ \\
\hline ZP 9 & 1,22 & $\mathrm{a}$ & 20,63 & $\mathrm{a}$ \\
\hline ZP10 & 1,75 & $\mathrm{a}$ & 39,29 & $\mathrm{a}$ \\
\hline ZP11 & 1,39 & $\mathrm{a}$ & 27,89 & $\mathrm{a}$ \\
\hline ZP12 & 1,58 & $\mathrm{a}$ & 39,29 & $\mathrm{a}$ \\
\hline ZP13 & 1,29 & $\mathrm{a}$ & 25,51 & $\mathrm{a}$ \\
\hline ZP14 & 1,50 & $\mathrm{a}$ & 39,29 & $\mathrm{a}$ \\
\hline ZP15 & 1,67 & $\mathrm{a}$ & 33,33 & $\mathrm{a}$ \\
\hline ZP16 & 1,41 & $\mathrm{a}$ & 32,77 & $\mathrm{a}$ \\
\hline ZP17 & 1,50 & $\mathrm{a}$ & 21,43 & $\mathrm{a}$ \\
\hline Promedio & $\mathbf{1 , 3 7}$ & & $\mathbf{3 0 , 5 6}$ & \\
\hline DMS (Tuckey alfa $\mathbf{0 , 0 5 )}$ & $\mathbf{0 , 9 3}$ & $\mathbf{2 3 , 9 3}$ & \\
\hline
\end{tabular}




\section{Cuadro $\mathrm{N}^{\circ} 6$ \\ VALORES MEDIOS DE RECTITUD Y CALIDAD DE FUSTE POR PROGENIES Y DIFERENCIA MÍNIMA SIGNIFICATIVA (DMS)}

\begin{tabular}{|l|c|c|}
\hline \multicolumn{1}{|c|}{ Progenie } & \multicolumn{1}{|c|}{$\begin{array}{c}\text { Rectitud } \\
\text { Fuste } \\
(\mathbf{1 - 4 )}\end{array}$} & $\begin{array}{c}\text { Calidad } \\
\text { Fuste } \\
(\%)\end{array}$ \\
\hline Todas las progenies & $1-2,5$ a & $11,10-49,29 \quad$ a \\
\hline Promedio & $\mathbf{1 , 3 7}$ & $\mathbf{3 0 , 5 6}$ \\
\hline DMS (Tuckey alfa = 0,05$)$ & $\mathbf{1 , 9 5}$ & $\mathbf{5 0 , 2 4}$ \\
\hline
\end{tabular}

\section{DISCUSIÓN}

\section{Crecimiento y Supervivencia}

$72 \%$.

La supervivencia del ensayo de coihue de Huillilemu a los 15 años de edad alcanzó un

Como referencia, plantaciones de coihue establecidas en la Cordillera de la Costa de Valdivia exhiben una supervivencia de $75 \%$ a $96 \%$ después de un año (Büchner, 2007); en plantaciones de cuatro años Alvarez y Lara (2008) indican supervivencia de 90 a $100 \%$; Betancurt (2015) indica supervivencia de 68,9\% para plantación de seis años establecida en LLancahue (Provincia de Valdivia); para otra plantación en la zona de Corral se informa una supervivencia de $86 \%$ a los siete años de edad (Donoso y Soto, 2010).

En cuanto a crecimiento, los IMA de altura $(1,03 \mathrm{~m})$ y de DAP $(1,2 \mathrm{~cm})$ obtenidos en el ensayo evaluado se comparan favorablemente con los reportados para renovales de coihue, los que fluctúan entre 0,2 y $0,8 \mathrm{~m}$ para la altura y 0,3 y $0,8 \mathrm{~cm}$ para el DAP (Cuadro $\mathrm{N}^{\circ} 7$ ). esta especie.

Esta situación es normal al comparar crecimiento de plantaciones y bosques naturales de

Al respecto, debe tenerse en cuenta que el ensayo evaluado posee 15 años, edad que coincide con la de máximos incremento medios anuales, que según autores citados por Donoso et al. (2007) ocurriría entre los 10 y 15 años para plantaciones de coihue y entre los 15 y 30 años para sus renovales. Estos rangos de edad son coincidentes con los registrados en el Cuadro $\mathrm{N}^{\circ} 7$ para efectos de comparación.

Por otra parte, los crecimientos obtenidos en el ensayo Huillilemu resultan de la misma magnitud que los señalados por distintos autores para plantaciones de coihue de edad y localidades similares a la del ensayo evaluado (Cuadro $\mathrm{N}^{\circ} 7$ ).

En general, aunque se han descrito crecimientos bastantes heterogéneos se reconoce que coihue manifiesta un crecimiento más bien rápido entre las especies arbóreas del cono sur (Cruz-Johnson, 2013). 
Cuadro $\mathrm{N}^{\circ} 7$

VALORES REFERENCIALES DE CRECIMIENTO DE COIHUE EN PLANTACIONES Y RENOVALES

\begin{tabular}{|c|c|c|c|c|}
\hline \multirow[b]{2}{*}{ Tipo de Formación } & \multicolumn{3}{|c|}{ Incremento Medio Anual } & \multirow[b]{2}{*}{ Fuente } \\
\hline & $\begin{array}{l}\text { Edad } \\
\text { (años) }\end{array}$ & $\begin{array}{l}\text { DAP } \\
(\mathrm{cm})\end{array}$ & $\begin{array}{l}\text { Altura } \\
\text { (m) }\end{array}$ & \\
\hline Renoval XI & $25-35$ & $0,3-0,7$ & $0,44-0,68$ & \multirow{2}{*}{$\begin{array}{l}\text { Delgado (1986 cit por Donoso et al., } \\
\text { 1999) }\end{array}$} \\
\hline Renoval VIII- IX & $25-46$ & $0,3-0,6$ & $0,25-0,55$ & \\
\hline Renoval Manejado & $30-40$ & $0,5-0,6$ & 0,48 & \multirow{3}{*}{ Donoso et al., 1999} \\
\hline Renoval Costa Valdivia & 27 & 0,47 & 0,62 & \\
\hline Renoval Andino Pilmaiquén & 23 & 0,43 & 0,44 & \\
\hline Renoval IX & 25 & $0,5-0,8$ & - & \multirow{2}{*}{$\begin{array}{l}\text { Donoso (1993 cit. por Cruz-Johnson, } \\
\text { 2013) }\end{array}$} \\
\hline Renoval X & $10-80$ & - & $0,2-0,8$ & \\
\hline Plantación Corral & 7 & 1,95 & 1,52 & Donoso y Soto, 2010 \\
\hline $\begin{array}{l}\text { Plantación Cordillera Costa } \\
\text { Valdivia }\end{array}$ & 1 & - & - & Büchner, 2007 \\
\hline Plantación Prov. Valdivia & 2 & - & 1,0 & Donoso et al., 2007 \\
\hline \multirow{3}{*}{ Plantación Frutillar } & 4 & - & 0.64 & \multirow{3}{*}{ Vita (1997 cit por Betancurt, 2015) } \\
\hline & 9 & 1,1 & 0,51 & \\
\hline & 14 & 1,9 & 0,62 & \\
\hline Plantación Valdivia & 3 & - & $0,9-1,25$ & $\begin{array}{l}\text { Donoso et al. (1986 cit por } \\
\text { Betancurt, 2015) }\end{array}$ \\
\hline Plantación Valdivia & 18 & 0,97 & 1,1 & \multirow{2}{*}{$\begin{array}{l}\text { Maureira (1995 cit por Betancurt, } \\
\text { 2015) }\end{array}$} \\
\hline Plantación Valdivia & 9 & $0,98-1,1$ & $1,1-1,2$ & \\
\hline Plantación Valdivia & 4 & 1,1 & 0,6 & $\begin{array}{l}\text { Alvarez y Lara (2008 cit por } \\
\text { Betancurt, 2015) }\end{array}$ \\
\hline Plantación con Manejo & 6 & 1,6 & 1,52 & $\begin{array}{l}\text { Soto y Ríos (2009 cit por Betancurt, } \\
2015\end{array}$ \\
\hline $\begin{array}{l}\text { Ensayo Huillilemu } \\
\text { Promedio }\end{array}$ & \multirow{3}{*}{15} & 1,2 & 1,03 & \\
\hline Mejor Familia & & 1,6 & 1,33 & \\
\hline $\begin{array}{l}\text { Mejor Zona de } \\
\text { Procedencia }\end{array}$ & & 1,3 & 1,12 & \\
\hline
\end{tabular}

(Fuente: Elaboración propia en base a documentos citados)

\section{Rectitud de Fuste}

Los individuos del ensayo presentan pobre calidad de fustes, con una muy escasa presencia de árboles rectos; el 97,9\% de ellos se concentran en las categorías 1 y 2 (menos rectas) y solo el $2,8 \%$ en las clases más rectas (3 y 4 ).

En el Cuadro $\mathrm{N}^{\circ} 8$ se comparan los resultados obtenidos en este estudio con los obtenidos por Betancurt (2015) en una plantación de coihue de 6 años de edad, los resultados evidencian una tendencia distinta, pero coinciden en identificar pocos árboles rectos. 


\section{Cuadro $\mathrm{N}^{\circ} 8$ \\ DISTRIBUCIÓN PORCENTUAL DE LOS ÁRBOLES EN CUATRO CATEGORÍAS DE RECTITUD}

\begin{tabular}{|l|r|r|r|r|}
\hline Fuente & \multicolumn{1}{|c|}{$\begin{array}{c}\text { 1 } \\
\text { - recto }\end{array}$} & \multicolumn{1}{c|}{$\mathbf{2}$} & \multicolumn{1}{c|}{$\mathbf{3}$} & \multicolumn{1}{|c|}{$\begin{array}{c}\mathbf{4} \\
\text { +recto }\end{array}$} \\
\hline $\begin{array}{l}\text { Plantación 6 años } \\
\text { (Betancurt, 2015) }\end{array}$ & 5,0 & 48,0 & 43,0 & 4,0 \\
\hline $\begin{array}{l}\text { Huillilemu, 15 años } \\
\text { (Este estudio) }\end{array}$ & 65,9 & 31,3 & 2,5 & 0,3 \\
\hline
\end{tabular}

'Los datos originales de Betancurt (2015) consideraban una escala de 3 categorías, y fueron modificados para hacerlos comparables con la clasificación en cuatro categorías empleadas en este estudio.

De acuerdo con los antecedentes bibliográficos las plantaciones más densas (1,5 x 1,5 m o $2 \times 2 \mathrm{~m}$ ) tendrían mejor forma de fuste que las de menor densidad $(2 \times 3 \mathrm{~m})$. En el caso el ensayo evaluado la densidad nominal entre individuos de coihue es de $2 \times 4 \mathrm{~m}$, pero atendiendo a que existe una plantación de raulí entre hileras, la densidad definitiva es de $2 \times 2 \mathrm{~m}$, aun así siendo un masivo relativamente denso, la rectitud del fuste sigue siendo deficiente.

Complementariamente, al comparar el crecimiento de coihue en claros y a campo abierto Wienstroer et al. (2003) concluyen que en el segundo caso se produce mayor crecimiento, pero que la mejor forma se produce en los claros, posiblemente como consecuencia de la competencia por luz.

\section{Bifurcaciones y Flechas Múltiples}

En el ensayo evaluado se observó una alta incidencia de individuos bifurcados (47,8\%) y con múltiple flecha $(24,9 \%)$, situación que es común en la especie. Soto y Ríos (2009) mencionan un porcentaje de bifurcación muy similar $(45,7 \%)$ para plantaciones de coihue de seis años establecidas en la precordillera de la costa de Valdivia, mientras que Betancurt (2015) informa de un $59 \%$ de bifurcaciones en plantaciones de la misma edad efectuadas en Llancahue (cercanías de Valdivia).

Por su parte, Donoso et al. (2015; cit. por Betancurt, 2015) indican entre un 40 y $50 \%$ de árboles bifurcados en plantaciones de coihue de tres años de edad en la región de Los Ríos, y que estas aumentarían hasta el 50 a $70 \%$ al cuarto año. Incluso tras efectuar podas de formación ${ }^{15}$ sigue existiendo una fuerte tendencia a la bifurcación, la que según Viñuales (2012) alcanza hasta el 45 a $53 \%$ de los árboles después de tres años de efectuada esta intervención.

En el Cuadro $N^{\circ} 9$ se presenta una comparación de las frecuencias relativas de los árboles bifurcados cuantificados en este estudio y en un trabajo de referencia (Betancurt, 2015). En él se observa que en ambos casos los porcentajes de árboles sin bifurcaciones y de árboles bifurcados son de la misma magnitud, sin embargo hay diferencias en la altura a la que estas se presentan.

\footnotetext{
${ }^{15}$ Eliminación de dobles o múltiples flechas y de ramas de crecimiento excesivo en detrimento del fuste principal
} 


\section{Cuadro $\mathrm{N}^{\circ} 9$ \\ DISTRIBUCIÓN PORCENTUAL DE LOS ÁRBOLES SEGÚN PRESENCIA Y ALTURA DE BIFURCACIONES}

\begin{tabular}{|l|r|r|r|r|}
\hline \multirow{2}{*}{ Fuente } & \multicolumn{1}{|c|}{$\begin{array}{c}\text { No } \\
\text { Bifurcados } \\
\text { (\%) }\end{array}$} & \multicolumn{3}{|c|}{$\begin{array}{c}\text { Bifurcados } \\
\text { (\%) }\end{array}$} \\
\cline { 3 - 5 } & & Sobre el DAP & Bajo el DAP & Total \\
\hline $\begin{array}{l}\text { Plantación 6 años } \\
\text { (Betancurt, 2015) }\end{array}$ & 41 & 11 & 48 & 59 \\
\hline $\begin{array}{l}\text { Huillilemu, 15 años } \\
\text { (Este estudio) }\end{array}$ & 52 & 33 & 15 & 48 \\
\hline
\end{tabular}

La alta incidencia de las bifurcaciones obedecería a que coihue presenta yemas preformadas sin catáfilos de protección y solo cubiertas por las estípulas de las hojas, en consecuencia es muy frecuentemente que las yemas terminales de los brotes aborten y que el tronco se construya a partir de las yemas laterales ubicada en la parte distal de cada brote, aumentando la frecuencia de bifurcaciones (Donoso et al., 2006).

Existen también otras variables que influyen en la forma del fuste de los individuos que generan bifurcación; una deficiente calidad de la planta al momento del establecimiento, expresado en el desequilibrio entre la parte área y radical, influye en el vigor de los brotes, generando malformaciones, por lo mismo, es importante evitar el daño a la yema terminal de las plantas.

\section{CONCLUSIONES}

El crecimiento en altura y diámetro del ensayo de coihue Huillilemu se encuentra dentro de los parámetros esperables. Existe poca diferenciación entre progenies, destacándose una de ellas que exhibe mejor crecimiento en altura (progenie 142) y 3 con mejor crecimiento en diámetro (progenies 261, 142 y 372). Por el contrario, no se detectan diferencias significativas de crecimiento entre zonas de procedencia.

En lo que respecta a atributos de calidad del fuste, no existen diferencias entre progenies ni entre zonas de procedencia, aun así existen algunos escasos árboles individuales que exhiben un adecuado comportamiento en estos atributos y que pueden ser de interés como progenitores en programas de mejoramiento genético.

Las deficiencias observadas en la calidad de fuste corresponden a una característica intrínseca de la especie y constituyen un desincentivo para su uso en plantaciones. No obstante, atendiendo a sus atractivas tasas de crecimiento, resulta de interés desarrollar iniciativas de mejoramiento genético para superar esta limitación y disponer así de una alternativa para contribuir a la diversificación de las plantaciones forestales.

\section{REFERENCIAS}

Álvarez, C. y Lara, A., 2008. Crecimiento de una plantación joven en fajas con especies nativas en la Cordillera de Los Andes de la provincia de Valdivia. Bosque Nativo 29 (3): 181-191.

Betancurt, G., 2015. Evaluación del desempeño de una plantación joven de coihue establecida a campo abierto en el predio Llancahue, comuna de Valdivia. Trabajo de Titulación presentado como parte de los requisitos para 
optar al Título de Ingeniero Forestal. Facultad de Ciencias Forestales y Recursos Naturales. Universidad Austral de Chile. Valdivia. 61 p.

Büchner, C., 2007. Respuesta inicial de una plantación de Nothofagus dombeyi (Mirb) Oerst a distintas dosis de fertilizante, en la precordillera de la Costa de Valdivia. Tesis Escuela de Ingeniería Forestal, Facultad de Ciencias Forestales, Universidad Austral de Chile. Valdivia, Chile.

En: http://cybertesis.uach.cl/tesis/uach/2007/fifb919r/doc/fifb919r.pdf. (Consulta junio, 2017).

Cruz-Johnson, P., 2013. Aplicación de relaciones de densidad-diámetro y de área potencial aprovechable en bosques de Nothofagus dombeyi (Mirb.) Oerst, como herramienta de planificación silvícola. Tesis doctoral Departamento de Ciencia y Tecnología Agroforestal y Genética. Universidad de Castilla La Mancha. Albacete, España. 120 p.

Donoso, C.; Donoso, P.; González, M. y Sandoval, V., 1999. Los bosques siempreverdes. Silvicultura de los Bosques Nativos de Chile. Editorial Universitaria, Santiago, Chile. p. 261-289.

Donoso, P.; Donoso, C.; Marchelli, P.; Gallo, L. y Escobar, B., 2006. Las especies arbóreas de los bosques templados de Chile y Argentina. Autoecología. Santiago, Chile. Marisa Cúneo Ediciones. p. 448- 461.

Donoso, P.; Soto, D.; Donoso, C. y Rivera, H., 2007. Crecimiento inicial en altura de plantaciones de coihue, roble y raulí en altitudes medias en la provincia de Valdivia, Chile. Bosque Nativo $N^{\circ}$ 40. Dic 2006 - Abr 2007. Pp: 8-12.

Donoso, P. y Soto, D., 2010. Plantaciones con especies nativas en el centro-sur de Chile: Experiencias, desafíos y oportunidades. Bosque Nativo $N^{\circ} 47$, julio-octubre 2010, pp: 10-17.

Quiroz, I. y Gutiérrez, B., 2014. Definición de zonas de procedencia para especies del bosque nativo. En: Propuesta de reglamento para semillas y plantas forestales. INFOR-SAG. Concepción, Chile. 74 p.

Soto, D. y Ríos, A., 2009. Seis años de respuesta a una plantación mixta de Coihue y Raulí en la Precordillera de la Costa de Valdivia. Bosque Nativo 44: 7-11.

Viñuales, M., 2012. Efecto de la poda temprana sobre el crecimiento y la forma en plantaciones jóvenes de Coihue y Raulí. Tesis Ingeniero Forestal. Valdivia, Chile. Facultad de Ciencias Forestales y Recursos Naturales, Universidad Austral de Chile. $39 \mathrm{p}$.

Wienstroer, M.; Siebert, H. y Müller-Using, B., 2003. Competencia entre tres especies de Nothofagus y Pseudotsogu menziesii en plantaciones mixtas jóvenes. Establecidas en la Precordillera andina de Valdivia. Bosque 24 (3): 17-30. 